\title{
Sensitization of rhTRAIL-resistant Triple-negative Breast Carcinoma Through Silibinin Co-Treatment
}

\author{
JASMINE M. MANOUCHEHRI ${ }^{1,2}$ and MICHAEL KALAFATIS ${ }^{1,2,3}$ \\ ${ }^{1}$ Department of Chemistry, Science and Research Center, Cleveland State University, Cleveland, OH, U.S.A.; \\ ${ }^{2}$ Center for Gene Regulation in Health and Disease (GRHD), Cleveland State University, Cleveland, OH, U.S.A.; \\ ${ }^{3}$ Department of Molecular Cardiology, Lerner Research Institute, Cleveland Clinic Foundation, Cleveland, OH, U.S.A.
}

\begin{abstract}
Background/Aim: Triple-negative breast cancer (TNBC) is the most fatal form of breast cancer due to the shortcomings of therapies. However, recombinant human tumor necrosis factor-related apoptosis-inducing ligand (rhTRAIL) is a promising anticancer therapeutic that possesses the capability to promote the induction of apoptosis in cancer cells, but some TNBCs are resistant to rhTRAIL's pro-apoptotic effects. Therefore, a combinatorial treatment approach with silibinin and rhTRAIL was considered in order to sensitize rhTRAIL-resistant TNBCs. Materials and Methods: The co-treatment of rhTRAIL and silibinin's impact on apoptosis induction in rhTRAIL-resistant TNBC BT-20 and HCC1937 cells was inspected via application of Annexin V/PI assays and western blot analysis. Results: Silibinin possessed the ability to sensitize the examined rhTRAIL-resistant TNBC cells to rhTRAIL-induced apoptosis through the up-regulation of death receptors 4 and 5 and the down-regulation of survivin transcriptionally. Conclusion: Silibinin is a good sensitizing agent for rhTRAIL-resistant TNBCs.
\end{abstract}

Triple negative breast cancers (TNBCs) are the most severe form of breast cancer carcinoma (1). TNBCs do not express estrogen receptor (ER), progesterone receptor (PR), and human epidermal growth factor receptor-2 (HER2), so traditional hormone-targeted therapies for breast cancer cannot be applied. Developing and examining therapeutics against

This Article is freely accessible online.

Correspondence to: Michael Kalafatis, Department of Chemistry, Cleveland State University, 2351 Euclid Avenue, Science and Research Center SR370, Cleveland, Ohio 44115, U.S.A. Tel: +1 2166872460/2467, Fax: +1 2166879298, e-mail: m.kalafatis@ csuohio.edu

Key Words: Apoptosis, breast cancer, survivin, death receptors, silibinin, rhTRAIL.
TNBCs is more problematic due to the absence of targeted molecular-oriented therapies resulting in worse clinical outcomes; hence, there is scientific interest in discovering and applying non-hormone related targeted therapies (1).

A potentially significant anticancer therapeutic is recombinant human tumor necrosis factor (TNF)-related apoptosis-inducing ligand (rhTRAIL), the optimized form of the endogenous death ligand TRAIL. rhTRAIL consists of the extracellular C-terminus of TRAIL amino acids 114281 lacking exogenous sequence tags (2-6). The appealing aspect of rhTRAIL is that it possesses the capacity to induce apoptosis in cancer cells without producing a toxic impact on normal, non-transformed cells (2-6). The extrinsic pathway of apoptosis is induced when rhTRAIL interacts with the extracellular death receptors (DRs) 4 and 5 stimulating the trimerization of the receptors, and consequently leading to activation of caspase 8 , activation of the executioner caspases 3,6, and 7, and the ultimate cleavage of poly (ADP-ribose) polymerase or PARP (hallmark of apoptosis) (7). Nonetheless, the pitfall of rhTRAIL treatment is apoptotic resistance detected in a majority of breast cancer cells due to the up-regulation of anti-apoptotic proteins such as survivin and the downregulation of DRs. Some phase I clinical trials were completed with rhTRAIL, but further trials were terminated due to a limited therapeutic efficacy (2). In response, many investigations have concentrated on alternative combinatorial approaches to combat resistance by assessing the abilities of various "mother-nature"-derived therapeutic agents to augment rhTRAIL-induced apoptosis in rhTRAILresistant cancers.

Silibinin is a flavonolignan derived from the seeds of Silybum marianum (milk thistle) $(8,9)$. Silibinin has been deemed safe for human use and been sold as a dietary supplement $(9,10)$. Silibinin has exhibited anti-cancer characteristics in various cancer cells including colon, lung, prostate, skin, hepatocellular, and breast cancer (11). In vitro, silibinin has been shown to promote apoptosis in hepatocellular, prostate, and breast cancers 
through activation of caspase 8 , subsequent activation of caspase 3 , and the cleavage of PARP; in addition, silibinininduced apoptosis occurred through the release of cytochrome c from the mitochondria facilitating caspase 9 activation, caspase 3 activation, and eventual PARP cleavage (8). Silibinin up-regulated DR4 and DR5 in colon cancer cells $(8,12,13)$. Silibinin decreased survivin expression in laryngeal squamous cell cancer cells and glioma cancer cells; silibinin downregulated c-FLIP expression in human glioma cells $(14,15)$. Silibinin decreased hepatocellular and lung carcinoma xenografts in vivo $(16,17)$. All these are encouraging results that make silibinin a safe and promising candidate for cotreatment with rhTRAIL.

This investigation focused on evaluating the effectiveness of silibinin as a sensitizing agent for rhTRAIL-resistant TNBC BT-20 and HCC1937 cells and elucidating the underlying mechanism for silibinin's sensitization. Through experimentation, we determined that silibinin retained the capability to decrease survivin expression and increase the expression of DRs in TNBC cells and thus, boosting rhTRAIL sensitivity in TNBC cells. Therefore, the presented evidence proposes that silibinin is an effective sensitizer for rhTRAIL-resistant TNBCs.

\section{Materials and Methods}

Drugs and chemicals. rhTRAIL was produced according to well defined and previously detailed protocols (3-5). Silibinin (molecular weight of $482.44 \mathrm{~g} / \mathrm{mol}$, Sigma, Milwaukee WI, USA) was dissolved in Polyethylene Glycol (PEG) molecular weight 400 (Fisher Scientific, Pittsburgh PA, USA) at $10 \mathrm{mg} / \mathrm{mL}$.

Cell culture. Human breast cancer BT-20 (ATCC HTB-19) cells were cultured in DMEM (Cleveland Clinic Cell Services Media Core, Cleveland OH, USA) supplemented with 10\% FBS (Gibco, Gaithersburg MD, USA), 1\% antibiotics-antimycotics (Gibco, Gaithersburg MD, USA), 1\% L-glutamine (Gibco, Gaithersburg MD, USA), $1 \%$ non-essential amino acids (Gibco, Gaithersburg MD, USA), and $1 \%$ sodium pyruvate (Gibco, Gaithersburg MD, USA). Human breast cancer HCC1937 (ATCC CRL-2336) cells were cultured in RPMI (Cleveland Clinic Cell Services Media Core) supplemented with 10\% FBS (Gibco, Gaithersburg MD, USA), $1 \%$ antibiotics-antimycotics (Gibco, Gaithersburg MD, USA), and 1\% L-glutamine (Gibco, Gaithersburg MD, USA). Human nontumorigenic breast epithelial MCF-10A (ATCC CRL-10317) cells were cultured in HuMEC ready medium (Gibco, Gaithersburg MD, USA). Cells were maintained in a humidified atmosphere with $5 \%$ $\mathrm{CO}_{2}$ at $37^{\circ} \mathrm{C}$. Cells were treated with drugs $24 \mathrm{~h}$ after plating, incubated with drugs for a further $72 \mathrm{~h}$, and collected for the various analyses detailed below.

Annexin V/PI assays-flow cytometry. Apoptosis was detected on the FACSCanto II after incubation with Annexin V-FITC and propidium iodide (PI) solution by following the procedure described by the manufacturer (Annexin V- FITC Apoptosis Detection Kit I, BD, sparks MD, USA). Analyses were completed by applying FACSDiva software and Flowing Software 2.
Assessing apoptotic associated protein levels. Cells were collected and total cell lysates were prepared according to previously published protocols (6). Aliquots of $35 \mu \mathrm{g}$ of protein were prepared and separated on $12 \%$ sodium dodecyl sulfate (SDS)polyacrylamide gels after denaturation with $4 \mathrm{X}$ Laemmli sample buffer (250 mM Tris-HCl, 8\% SDS, 40\% glycerol, 8\% BME, and $0.02 \%$ Bromophenol Blue). After proteins were transferred to PVDF membranes (Millipore, Temecula CA, USA) via the semi-dry transfer method (Bio-Rad, Richmond CA, USA), the membranes were blocked with $5 \%$ non-fat milk and incubated overnight at $4{ }^{\circ} \mathrm{C}$ with a diluted primary antibody: anti-PARP, anti-caspase 3 , anticleaved caspase 3 , anti-caspase 7 , anti-caspase 8 , anti-cleaved caspase 8 , or anti-survivin (Cell Signaling Technology, Danvers, MD, USA). The membranes were washed and developed as described previously (6).

Western blot analysis of DR4 and DR5 expression. Cells were collected and prepared according to previously published protocols (6). PVDF membranes were blocked, incubated overnight at $4^{\circ} \mathrm{C}$ with anti-DR4 or anti-DR5 (Cell Signaling Technology, Danvers MD, USA), washed, and developed as above.

Flow cytometry analysis of DR4 and DR5 expression. Cells were collected, prepared, and incubated with anti-DR4-PE, anti-DR5PE or a mouse IgG1 kappa isotype control (eBioscience, Pittsburgh PA, USA) according to previously published protocols (6); the isotype control was used to compensate for any nonspecific binding. DR4 and DR5 membrane expressions were analyzed on a BD FACSCanto II flow cytometer applying FACSDiva software.

Reverse Transcription-PCR analysis for DR4, DR5, and survinin. Total RNA was extracted from cells using TRIzol reagent (Ambion, Pittsburgh PA, USA). Reverse transcription polymerase chain reaction (RT-PCR) was performed following the manufacturer's protocol (Invitrogen SuperScript III One-Step RT-PCR System with Platinum ${ }^{\circledR}$ Taq DNA Polymerase, Ambion, Pittsburgh PA, USA). Human DR5 mRNA was amplified using the forward primer 5'GGGAGCCGCTCATGAGGAAGTTGG-3' and the reverse primer 5'-GGCAAGTCTCTCTCCCAGGTCTC-3'. Human DR4 mRNA was amplified using the forward primer 5'-GAGCAACGCAGACTCGCT-3' and the reverse primer 5'- TCACTCCAAGGACACGGC-3'. For survivin, forward primer 5'- CAAGGACCACCGCATCT-CTAC-3' and the reverse primer 5'-AGTCTGGCTCGTTCTCAGTGG-3' were used. For $\beta$-actin, the forward primer 5'TGACGGGGTCACCCACACTGTGCC-3' and the reverse primer 5'-CTGCATCCTGTCGGCAATGCCAG-3' were used. cDNA synthesis was performed at $60^{\circ} \mathrm{C}$ for 30 min using the Applied Biosystems GeneAmp PCR System 9700. The PCR cycling conditions (40 cycles) were chosen as follows: denature for $2 \mathrm{~min}$ at $94^{\circ} \mathrm{C}$, anneal for $30 \mathrm{sec}$ at $55^{\circ} \mathrm{C}$ for survinin and DR4 and $65^{\circ} \mathrm{C}$ for DR5 and $\beta$-actin, extend for $1 \mathrm{~min}$ and $30 \mathrm{sec}$ at $68^{\circ} \mathrm{C}$, and execute a final extension for $10 \mathrm{~min}$ at $68^{\circ} \mathrm{C}$. Reaction products were analyzed on $1.2 \%$ agarose gels. The bands were visualized by ethidium bromide (Invitrogen, Carlsbad CA, USA) and an UV illuminator (UVP).

Statistical analysis. Data were analyzed using the Student's $t$-test, and the differences between experimental and control groups were deemed statistically significant at $p$-values less than 0.05 

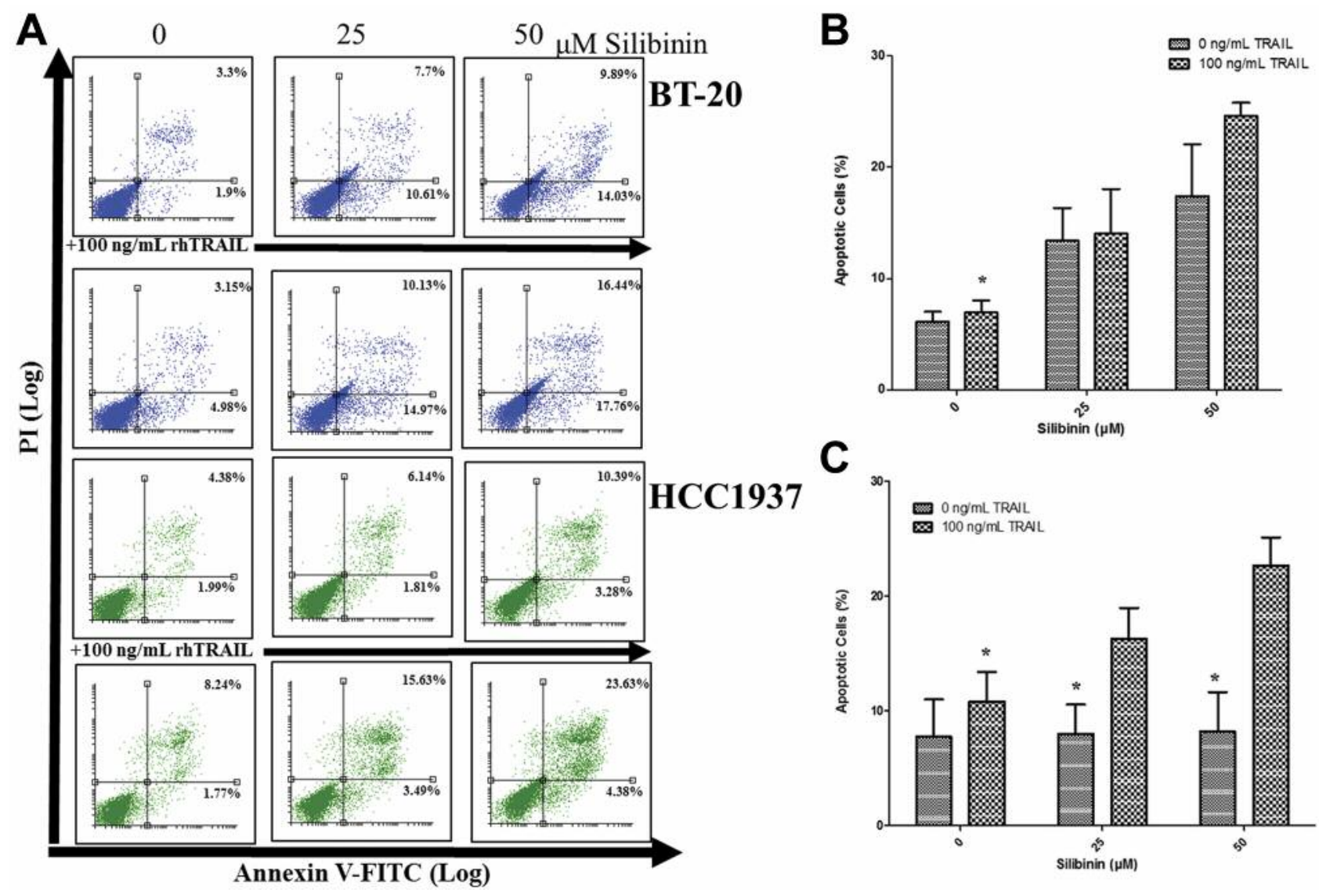

Figure 1. Silibinin augments rhTRAIL-induced apoptosis in breast cancer cells. Cells were treated with silibinin in the presence and absence of 100 $n g / m L$ rhTRAIL for 72 hours. (A) For the representative FACS plots, the left top quadrants represent Annexin $V^{-} / P^{+}$-dead cells, the right top quadrants represent Annexin $V^{+} / P I^{+}$-late apoptotic, dead cells, the left bottom quadrants represent Annexin $V^{-} / P I^{-}$-viable cells, and the right bottom quadrants represent Annexin $V^{+} / P I^{-}$-early apoptotic cells. Bar graphs (B) BT-20 and (C) HCC1937 display the average total percent of apoptotic cells (Annexin $V^{+}$cells) $\pm S E M$ calculated from three independent experiments carried out in triplicate $(n=9)$ ). $p<0.05$ except* .

\section{Results}

Evaluating rhTRAIL-induced apoptosis through FACS analysis. FACS analysis was conducted on TNBC cells treated with 25 or $50 \mu \mathrm{M}$ silibinin in the presence or absence of $100 \mathrm{ng} / \mathrm{ml}$ rhTRAIL for 72 hours to determine silibinin's sensitizing effects on rhTRAIL-induced apoptosis (Figure 1). Silibinin augmented rhTRAIL-induced apoptosis in both TNBC cell lines. For example, TNBC BT-20 and HCC 1937 cells treated with $50 \mu \mathrm{M}$ of silibinin generated on average about $15 \%$ and $10 \%$ apoptotic cells, respectively $(p<0.05)$; yet, the co-treatment of $50 \mu \mathrm{M} \mathrm{Q}$ and $100 \mathrm{ng} / \mathrm{ml}$ rhTRAIL on BT-20 and HCC1937 cells generated on average about $25 \%$ apoptotic cells $(p<0.05)$. In addition, it should be pointed out that the single agent treatment of 100 $\mathrm{ng} / \mathrm{ml}$ rhTRAIL did not generate a significant amount of apoptotic TNBC cells in comparison to the vehicle-treated control $(p>0.05)$.
Observing the induction of the pathway of apoptosis. TNBC cells were treated with the single agents of silibinin (25 and $50 \mu \mathrm{M})$ and rhTRAIL $(100 \mathrm{ng} / \mathrm{ml})$ along with the cotreatments for $72 \mathrm{~h}$. The expression of proteins caspase 8 , caspase 3, caspase 7, and cleaved PARP were all assessed in the treated cells, and the co-treatment of silibinin and rhTRAIL enhanced the expression of the examined apoptotic proteins in comparison to the vehicle-treated controls and single agent treatments (Figure 2). The data confirms that apoptosis was induced by the co-treatment through the extrinsic pathway as indicated by the activation of caspase 8 , the activation of executioner caspases 3 and 7 , and the cleavage of PARP. Furthermore, it should be recognized that PARP cleavage was not detected in rhTRAIL-treated TNBC cells; this aligns with other researchers' results that TNBC BT-20 and HCC1937 cells are rhTRAIL-resistant (18). Moreover, silibinin did not influence proliferation of nontumorigenic breast epithelial MCF-10A cells (19). In 

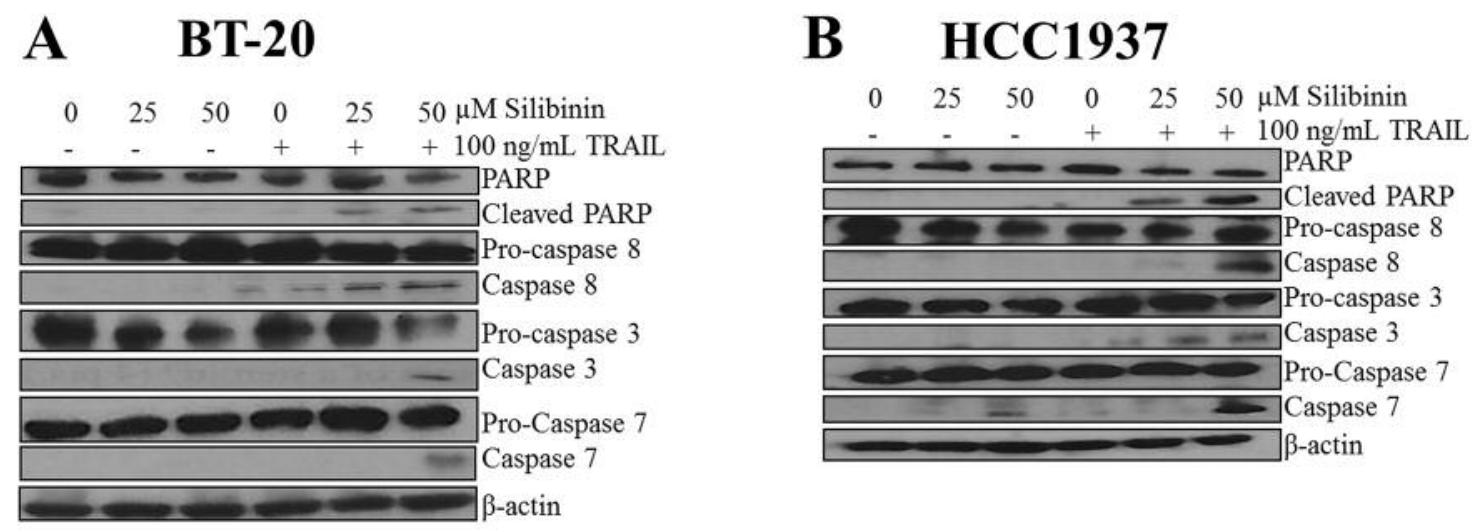

Figure 2. Silibinin and rhTRAIL trigger the induction of the extrinsic pathway of apoptosis. TNBC (A) BT-20 and (B) HCC1937 cells were sensitized to rhTRAIL-induced apoptosis through silibinin co-treatment as indicated by activation of caspase 8, activation of executioner caspases 3 and 7 , and PARP cleavage. $\beta$-Actin was used as loading control and probed for each blot; the $\beta$-actins shown are representative results for each cell line.

addition, TRAIL did not induce apoptosis in MCF-10A cells (20). This evidence supports the notion that the co-treatment of rhTRAIL and silibinin will impact breast cancer cells not normal, non-tumorigenic breast cells. Overall, these findings (Figure 2) in conjunction with the FACS analysis (Figure 1) indicate that silibinin bolsters the sensitization of rhTRAILresistant TNBC cells to rhTRAIL-induced apoptosis via the stimulation of the extrinsic pathway of apoptosis.

Silibinin's effects on DR4 and DR5 expression. Western blot and flow cytometry analyses were conducted to ascertain silibinin's effect on DR4 and DR5 protein and cell surface expression in TNBCs. Silibinin promoted the up-regulation of the protein expression of DR5 in TNBC cells in a dosedependent manner after 72 hours of treatment (Figure 3); in addition, silibinin significantly enhanced the protein expression of DR4 in HCC1937 $(p<0.05)$ but not in BT-20 cells. The FACS analysis of the cell surface expressions of DR4 and DR5 in TNBC cells treated with silibinin were in alignment with the increased protein expression of DR5 in TNBC cells and the increased protein expression of DR4 in only TNBC HCC1937 cells. Silibinin did not affect the expression of DRs in non-tumorigenic breast epithelial MCF-10A cells after 72 hours of treatment (Figure 3E). Thus, silibinin induces DR5 up-regulation in TNBC cells and DR4 up-regulation in TNBC HCC1937 cells; the upregulation of DRs by silibinin improves rhTRAIL sensitivity in these TNBC cells.

Silibinin's influence on survivin expression. Survivin is a member of the inhibitor of apoptosis (IAP) gene family; it is a short-lived protein that is overexpressed in cancer cells especially breast cancer $(21,22)$. Survivin acts as an inhibitor of cell death by preventing the proper execution of the extrinsic and intrinsic pathways of apoptosis, but the mechanism of this inhibition has not been fully elucidated $(21,22)$. Through western blot analysis, silibinin down-regulated survivin expression in a dose-dependent manner in TNBC cells and thereby, facilitating enhanced rhTRAIL sensitivity via the full execution of the extrinsic pathway of apoptosis (Figure 4A).

Silibinin's effect on DRs and survivin expression at the transcriptional level. Through analyses, silibinin demonstrated the ability to enhance the expression of DR5 in TNBC cells, enhance the expression of DR4 in TNBC HCC1937 cells, and diminish the expression of survivin in TNBC, so the next step was to assess if silibinin effects the expression of those apoptotic proteins transcriptionally. Thus, RT-PCR was carried out and $\beta$-actin was applied as a positive control (Figure 4B). The RT-PCR analyses revealed that silibinin did decrease survivin mRNA expression in both TNBC cells. In addition, silibinin did increase the expression of DR4 mRNA in HCC1937 cells but not in BT-20 cells; also, silibinin did increase the expression of DR5 mRNA in both TNBC cells. These results provide the mechanism of action by which silibinin influences rhTRAIL sensitivity in rhTRAIL-resistant TNBCs by effecting the expression of DRs and survivin at the transcriptional level.

\section{Discussion}

TNBC is a deadly form of breast carcinoma. rhTRAIL induces apoptosis through the extrinsic pathway and through the intrinsic pathway independent of p53 in cancer cells. On the other hand, traditional chemotherapy and radiation treatments are dependent on p53 for the full execution of the intrinsic pathway of apoptosis $(2-5,23,24)$. rhTRAIL is a more favorable therapeutic agent because many cancers have a non-functional TP53 gene that leads to necrosis over 
A

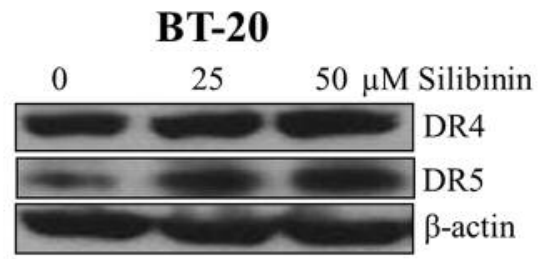

B

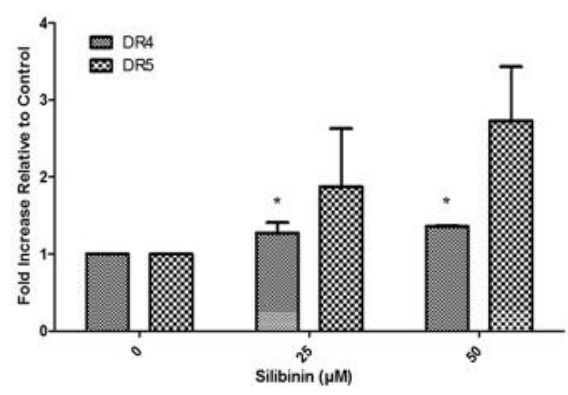

C

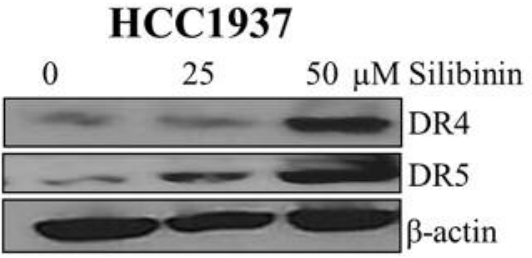

D

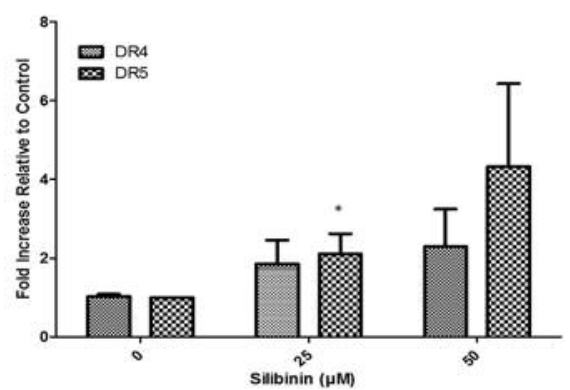

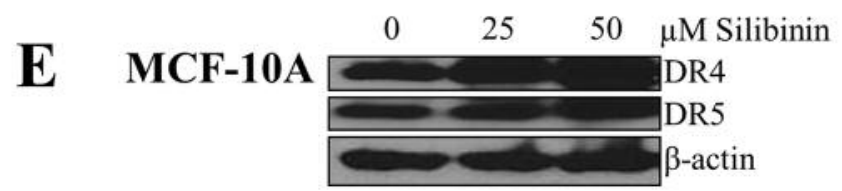

Figure 3. Expression of DRs in silibinin-treated TNBC cells. (A) DR4 and DR5 (mature form) protein levels were assessed in BT-20 cells; silibinin increased the expression of DR5 in BT-20 cells in a dose-dependent manner after 72 hours of treatment. (B) DR4 and DR5 cell surface expression levels for silibinin-treated BT-20 cells were analyzed by flow cytometry; the bar graphs represent the average fold increase in DR4 or DR5 cell surface expressions relative to the vehicle-treated control \pm SEM from three independent experiments performed in triplicate $(n=9)$. $p<0.05$ except*. (C) DR4 and DR5 (mature form) protein levels were analyzed in HCC1937 cells; silibinin increased the expression of DR4 and DR5 in HCC1937 cells. Cell surface expression of (D) DR4 and DR5 for silibinin-treated breast cancer HCC1937 were assessed by flow cytometry. (E) DR4 and DR5 (mature form) protein levels were assessed for MCF-10A cells treated with silibinin, and DR4 and DR5 expressions were not significantly altered.

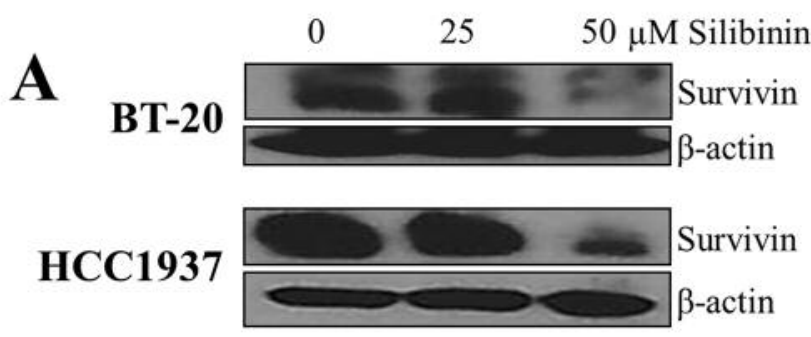

B

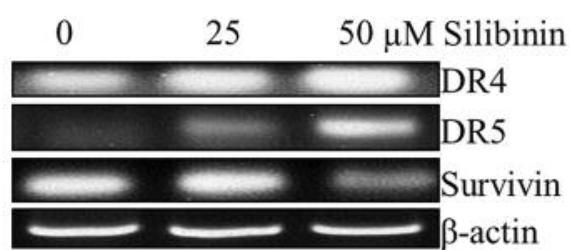

HCC1937

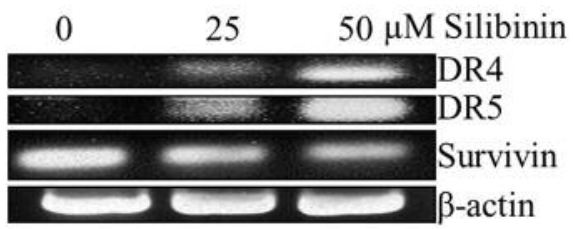

Figure 4. Silibinin's mechanism of sensitization occurs transcriptionally. (A) Western blotting detected that silibinin reduces the expression of the anti-apoptotic protein survivin in TNBC cells in a dose-dependent manner. (B) BT-20 and HCC1937 cells were treated with single agent silibinin for $72 \mathrm{~h}$ and RT-PCR was completed with $\beta$-actin as a positive control. Silibinin down-regulated survivin mRNA expression in TNBC cells, upregulated DR5 mRNA expression in TNBC cells, and up-regulated DR4 mRNA expression in HCC1937 cells. 
apoptosis, so if chemotherapy and radiation are applied, then negative side effects could be observed in patients. Despite this, there are factors that are implicated in resistance to rhTRAIL that lead to clinical trials being terminated due to the absence of clinical efficacy (25-28). Our study sought to combat rhTRAIL resistance in TNBC BT-20 and HCC1937 cells through the application of the "mother-nature"-derived silibinin as a sensitizing agent. In vitro examination, revealed that silibinin is an effective sensitizer for the rhTRAILresistant TNBC BT-20 and HCC1937 cells.

Western blot analysis and Annexin V/PI assays were executed in order to evaluate the consequences of the cotreatment of silibinin and rhTRAIL. Results gathered from both assays concluded that silibinin enhances rhTRAILinduced apoptosis in TNBC BT-20 and HCC1937 cells through the completion of the extrinsic pathway as presented by the augmented caspase 8 activation, executioner caspases 3 and 7 activation, and PARP cleavage (a hallmark of apoptosis). Consequently, the mechanism for silibinin's sensitization needed to be established.

Some rhTRAIL-resistant cancer cell lines have displayed low expression levels of DR4 and DR5, and some studies have postulated that this lack of expression of DRs hinders rhTRAIL from inducing apoptosis in those cancer cell lines $(25,28)$. Accordingly, this study inspected the protein and cell surface expressions of DR4 and DR5 in silibinin-treated TNBC cells via western blot and FACS analyses. From these analyses, we determined that silibinin induced the upregulation of DR5 in both TNBC cells and the up-regulation of DR4 in TNBC HCC1937 cells. In order to confirm that silibinin only affects DR4 and DR5 expression in cancer cell lines, we checked the expression of DRs in silibinin-treated non-tumorigenic breast epithelial MCF-10A cells and affirmed that silibinin did not impact the expression of either DR in those non-tumorigenic breast epithelial cells.

Additionally, silibinin's influence on the expression of the IAP survivin was probed for because survivin is expressed at high levels in breast cancer cells and interferes in the proper execution of the extrinsic pathway of apoptosis $(21,22)$. We discovered that silibinin significantly down-regulated the expression of survivin in both TNBC cells and thus aiding in intensifying rhTRAIL sensitivity in the examined TNBC cell lines. The underlying mechanism for this decrease in expression was then assessed through RT-PCR analysis.

Elucidation of silibinin's mechanism of sensitization was carried out through RT-PCR analysis of DR4, DR5, and survivin. Silibinin increased DR5 mRNA expression in both TNBC cell lines, silibinin increased DR4 mRNA expression in HCC1937, and silibinin decreased survivin mRNA expression in both TNBC cell lines. Therefore, silibinin enhances the expression of DR4 and DR5 and decreases survivin expression transcriptionally and thereby, boosting rhTRAIL sensitivity in TNBC cells.
The aim of this study was to optimize the therapeutic efficacy of rhTRAIL through the application of the "mothernature"-derived compound silibinin. Our findings indicate that silibinin was effective in overcoming the challenge of rhTRAIL-resistance in TNBC BT-20 and HCC1937 cells through up-regulation of DRs and down-regulation of survivin transcriptionally. In conclusion, silibinin is a potent sensitizing agent for rhTRAIL-resistant TNBCs.

\section{References}

1 Tomao F, Papa A, Zaccarelli E, Rossi L, Caruso D, Minozzi M, Vici P, Frati L and Tomao S: Triple-negative breast cancer: new perspectives for targeted therapies. OncoTargets and Thepary 8 : 177-193, 2015.

2 Kuijlen J, Bremer, Mooij J, Dunnen D and Helfrich: Review: On TRAIL for malignant glioma therapy? Neuropath Applied Neurobiol 36: 168-182, 2010.

3 Wang D and Shi L: High-level expression, purification, and in vitro refolding of soluble tumor necrosis factor-related apoptosisinducing ligand (TRAIL). Applied Biochem and Biotech 157: 19, 2009

4 Shen Y-L, Zhang Y, Sun A-Y, Xia X-X, Wei D-Z and Yang S-L: High-level production of soluble tumor necrosis factor-related apoptosis-inducing ligand (Apo2L/TRAIL) in high-density cultivation of recombinant Escherichia coli using a combined feeding strategy. Biotech Letters 26: 981-984, 2004.

5 Luo Q, Shen Y-L, Wei D-Z and Cao W: Optimization of culture on the overproduction of TRAIL in high-cell-density culture by recombinant Escherichia coli. Applied Microbio Biotech 71: 184-191, 2006.

6 Turner K, Lindner D and Kalafatis M: Recombinant human tumor necrosis factor-related apoptosis-inducing ligand selectively induces apoptosis in malignant melanoma. Int J Cancer Oncol 4: 1-8, 2017.

7 Fulda S and Debatin K-M: Extrinsic versus intrinsic apoptosis pathways in anticancer chemotherapy. Oncogene 25: 4798-4811, 2006.

8 Division J, Tiwari P, Mishra K and Division M: Silibinin in cancer therapy: a promising prospect. Cancer Res Frontiers 1: 303-318, 2015.

9 Deep G and Agarwal, R: Antimetastatic efficacy of silibinin: molecular mechanisms and therapeutic potential against cancer. Cancer Metastasis Rev 29: 447-463, 2010.

10 Hoh C, Boocock D, Marczylo T, Singh R, Berry D, Dennison A, Hemingway D, Miller A, West K, Euden S, Garcea G, Farmer P, Steward W and Gescher A: Pilot study of oral silibinin, a putative chemopreventive agent, in colorectal cancer patients: silibinin levels in plasma, colorectum, and liver and their pharmacodynamic consequences. Clin Cancer Res 12: 29442950, 2006.

11 Flaig T, Glodé M, Gustafson D, van Bokhoven A, Tao Y, Wilson S, Su L, Li Y, Harrison G, Agarwal R, Crawford D, Lucia S and Pollak M: A study of high-dose oral silybin-phytosome followed by prostatectomy in patients with localized prostate cancer. The Prostate 70: 848-855, 2010.

12 Kauntz H, Bousserouel S, Gossé F and Raul F: Silibinin triggers apoptotic signaling pathways and autophagic survival response in human colon adenocarcinoma cells and their derived metastatic cells. Apoptosis 16: 1042-1053, 2011. 
13 Kauntz H, Bousserouel S, Gossé F and Raul F: The flavonolignan silibinin potentiates TRAIL-induced apoptosis in human colon adenocarcinoma and in derived TRAIL-resistant metastatic cells. Apoptosis 17: 797-809, 2012.

14 Yang X, Li X, An L, Bai B and Chen J: Silibinin induced the apoptosis of Hep-2 cells via oxidative stress and downregulating survivin expression. Eur Arch OtoRhinoLaryngol 270: 2289-2297, 2013.

15 Son Y, Kim E, Kim J, Kim S, Kwon T, Yoon A-R, Yun C-O and Choi K: Silibinin sensitizes human glioma cells to TRAILmediated apoptosis via DR5 up-regulation and down-regulation of c-FLIP and survivin. Cancer Res 67: 8274-8284, 2007.

16 Cui W, Gu F and Hu K-Q: Effects and mechanisms of silibinin on human hepatocellular carcinoma xenografts in nude mice. World J Gastroenterol 15: 1943, 2009.

17 Singh R, Mallikarjuna, Sharma G, Dhanalakshmi S, Tyagi A, Chan D, Agarwal C and Agarwal R: Oral silibinin inhibits lung tumor growth in athymic nude mice and forms a novel chemocombination with doxorubicin targeting nuclear factor kappaB-mediated inducible chemoresistance. Clin Cancer Res 10: 8641-8647, 2004.

18 Rahman M, Pumphrey JG, Lipkowitz S: The TRAIL to targeted therapy of breast cancer. Adv Cancer Res 103: 43-73, 2009.

19 Jahanafrooz Z, Motameh N and Bakhshandeh B: Comparative evaluation of silibinin effects on cell cycling and apoptosis in human breast cancer MCF-7 and T47D cell lines. Asian Pac J Cancer Prev 17: 2661-2665, 2016.

20 Dong Y, Yin S, Li J, Jiang C, Ye M and Hu H: Bufadienolide compounds sensitize human breast cancer cells to TRAILinduced apoptosis via inhibition of STAT3/Mcl-1 pathway. Apoptosis 16: 394-403, 2011.

21 Ryan B, O'Donovan N and Duffy M: Survivin: a new target for anti-cancer therapy. Cancer Treat Rev 35: 553-562, 2009.
22 Altieri D: Survivin, versatile modulation of cell division and apoptosis in cancer. Oncogene 22: 8581-8589, 2003.

23 Liu J, Zhang C, Hu W and Feng Z: Tumor suppressor p53 and its mutants in cancer metabolism. Cancer Letters 356: 197-203, 2015.

24 Guan Y-S, He Q and Zou Q: Status quo of p53 in the treatment of tumors. Anti-Cancer Drugs 27: 811-818, 2016.

25 Jung Y-H, Heo J, Lee Y, Kwon T and Kim Y-H: Quercetin enhances TRAIL-induced apoptosis in prostate cancer cells via increased protein stability of death receptor 5. Life Sci 86: 351$357,2010$.

26 Psahoulia F, Drosopoulos K, Doubravska L, Andera L and Pintzas A: Quercetin enhances TRAIL-mediated apoptosis in colon cancer cells by inducing the accumulation of death receptors in lipid rafts. Mol Cancer Ther 6: 2591-2599, 2007.

27 Yi L, Zongyuan Y, Cheng G, Lingyun Z, GuiLian Y and Wei G: Quercetin enhances apoptotic effect of tumor necrosis factorrelated apoptosis-inducing ligand (TRAIL) in ovarian cancer cells through reactive oxygen species (ROS) mediated CCAAT enhancer-binding protein homologous protein (CHOP)-death receptor 5 pathway. Cancer Sci 105: 520-527, 2014.

28 Kim J, Kim E, Park S, Lim J, Kwon T and Choi K: Quercetin sensitizes human hepatoma cells to TRAIL-induced apoptosis via Sp1-mediated DR5 up-regulation and proteasome-mediated c-FLIPS down-regulation. J Cell Biochem 105: 1386-1398, 2008.
Received September 28, 2017

Revised October 12, 2017

Accepted October 13, 2017 